\title{
Validation of new TRUS biopsy techniques for PI-RADS 4 or 5
}

\section{Taein An, Byung Kwan Park}

Department of Radiology, Samsung Medical Center, Sungkyunkwan University School of Medicine, Seoul, Korea

Received: July 30, 2020

Revised: September 10, 2020

Accepted: September 11, 2020

Corresponding author:

Byung Kwan Park

Department of Radiology,

Samsung Medical Center,

Sungkyunkwan University

School of Medicine, 81

Irwon-ro, Gangnam-gu,

Seoul 06351, Korea

Tel: +82-2-3410-6457

E-mail: rapark@skku.edu

\section{ABSTRACT}

Purpose: Recently, new magnetic resonance imaging (MRI)-transrectal ultrasound (TRUS) techniques and imaging features of Prostate Imaging and Report and Data System (PI-RADS) 4 or 5 have been reported. The aim of this study was to validate the outcomes of new TRUS-guided biopsy techniques for cancer detection in patients with PIRADS 4 or 5.

Methods: Between June 2018 and November 2018, 94 men underwent TRUS-guided biopsy after PI-RADS $4(n=59)$ or $5(n=35)$ was categorized as an index lesion on MRI. For PI-RADS 4 group, target biopsy was performed in 5 and combination biopsy (target and systematic biopsies) was in 54. For PI-RADS 5 group, target biopsy was performed in 19 and combination biopsy was in 16 . Target to combination biopsy ratios and significant cancer detection rates (CDRs) were compared between the groups. Significant cancer was defined as a Gleason score $\geq 7$ tumor. Standard reference was biopsy examination. Fisher's exact were used for statistical analysis.

Results: Target to combination biopsy ratios was 5:54 in the PI-RADS 4 group and 19:16 in the PI-RADS 5 group $(P<0.0001)$. The significant CDR of the target biopsy were $42.4 \%$ $(25 / 59)$ in the PI-RADS 4 group and $82.6 \%(29 / 35)$ in the PI-RADS 5 group $(P=0.0002)$. The significant CDR of combination biopsy was $44.1 \%(26 / 59)$ in the PI-RADS 4 group and $85.7 \%(30 / 35)$ in the PI-RADS 5 group $(\mathrm{P}<0.0001)$.

Conclusion: New TRUS biopsy techniques provides high significant CDR patients with PI-RADS 4 or 5 . Therefore, TRUS should be performed prior to fusion or in-bore biopsy to determine if these categories are visible.

Keywords: Biopsy; Prostate; Ultrasonography
This is an Open Access article distributed under the terms of the Creative Commons Attribution Non-Commercial License (https:// creativecommons.org/licenses/ by-nc/4.0/).

\section{INTRODUCTION}

Prostate Imaging and Report and Data System version 2 (PI-RADSv2) has been introduced to use in the clinical practice since 2015 [1-3]. PI-RADSv2 categorizes prostate lesions using magnetic resonance imaging (MRI) features to stratify the risk of prostate cancer. Many studies have validated the utility of PI-RADSv2 in detecting significant cancer [4-9]. MRI-transrectal ultrasound (TRUS) fusion or in-bore MRI biopsy is usually performed to target PI-RADS 4 or 5 because these categories have so strong signal intensities on diffusion-weighted, T2-weighted, 
and dynamic contrast-enhanced MRI. PI-RADSv2 defines that these categories are likely or highly likely to have significant cancer [1-3].

TRUS is not used for lesion targeting because it is generally accepted that prostate cancer is not clearly seen on TRUS. However, it is still unclear if significant cancer is really invisible on TRUS. Recently, new TRUS techniques and imaging features have been reported to detect significant cancer in patients with PI-RADS 4 or 5 [10]. Only a few articles have introduced the new biopsy techniques to set up the new protocols of TRUS and difference of lesion location between MRI and TRUS [10-13]. Therefore, there is no validation study to show how cancer detection has been improved with these new TRUS features and biopsy. Our hypothesis was that these categories are visible on TRUS and targeted by TRUS due to high incidence of significant cancer. The aim of this study was to validate the outcomes of new TRUS-guided biopsy techniques for cancer detection in patients with PIRADS 4 or 5.

\section{METHODS}

\section{Patient selection}

Between June 2018 and November 2018, 166 men underwent TRUS-guided biopsy (Fig. 1). Of these patients, 72 were excluded because of the following criteria: PI-RADS 1-3 $(\mathrm{n}=64)$, low ( $<2.5 \mathrm{ng} / \mathrm{mL}$ ) prostate specific antigen (PSA; $n=7)$, and previous treatments $(n=1)$.
The remaining 94 men (median, 67.5 years; range, 40 to 87 ) met for the following inclusion criteria: high ( $\geq 2.5 \mathrm{ng} / \mathrm{mL}$ ) PSA, pre-biopsy MRI, and PI-RADS 4 or 5 . Their PSA, prostate volume, PSA density, MRI-biopsy interval, and tumor size ranged 2.8 to $2,783.6 \mathrm{ng} / \mathrm{mL}$ (median, 5.7), 12 to $304 \mathrm{~mL}$ (35), 0.04 to $108.73 \mathrm{ng} / \mathrm{mL} 2$ (0.17), 0 to 646 days (40), and 2.1- to $41.0 \mathrm{~mm}$ (12.0), respectively. These patients were divided into PI-RADS 4 group $(n=59)$ and PI-RADS 5 group $(n=35)$. Patient demographics in each group were summarized in the Table 1.

Table 1. Patient demographics

\begin{tabular}{lccc}
\hline \multirow{2}{*}{ Demographic } & \multicolumn{2}{c}{ TRUS-guided biopsy $(\mathrm{n}=94)$} & \multirow{2}{*}{ P-value } \\
\cline { 2 - 3 } & PI-RADS 4 (n=59) & PI-RADS 5 ( $=35)$ & \\
\hline Age (yr) & $64(40-82)$ & $71(57-87)$ & 0.0002 \\
PSA (ng/mL) & $4.9(2.8-30.3)$ & $7.8(2.9-2,783.6)$ & 0.0002 \\
$\begin{array}{c}\text { Prostate } \\
\text { volume (mL) }\end{array}$ & $34(12-162)$ & $36(16-304)$ & 0.9813 \\
$\begin{array}{c}\text { PSAD } \\
\left.\text { (ng/mL }{ }^{2}\right)\end{array}$ & $0.14(0.04-0.76)$ & $0.20(0.07-108.7)$ & 0.0037 \\
$\begin{array}{c}\text { MRI-biopsy } \\
\text { interval (day) }\end{array}$ & $46(8-646)$ & $30(0-73)$ & 0.0075 \\
$\begin{array}{c}\text { Tumor size } \\
(\mathrm{mm})\end{array}$ & $9.3(2.1-14.6)$ & $20.0(15.0-41.0)$ & $<0.0001$ \\
\hline
\end{tabular}

Values are presented as median (range).

TRUS, transrectal ultrasound; PI-RADS, Prostate Imaging Reporting and Data System; PSA, prostate specific antigen; PSAD, prostate specific antigen density; MRI, magnetic resonance imaging.

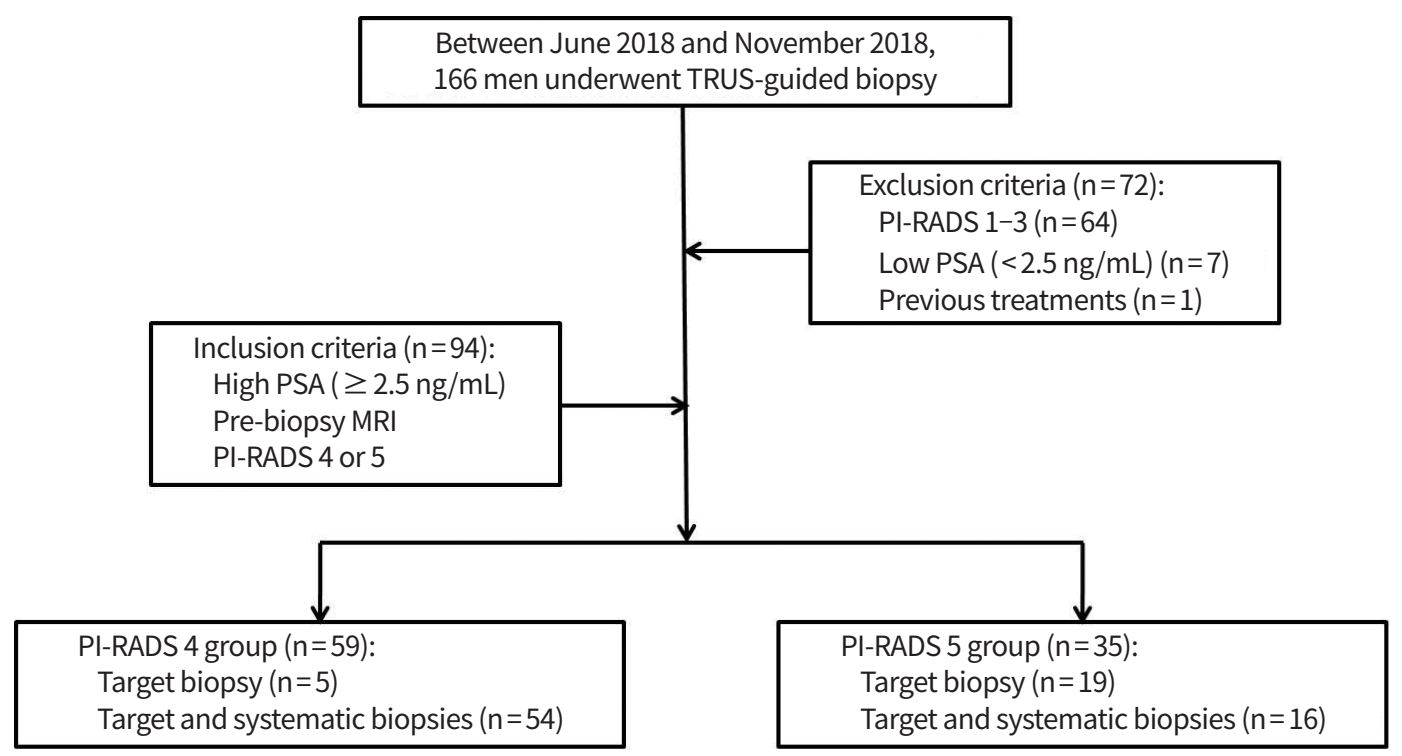

Fig. 1. Flow diagram for study population. TRUS, transrectal ultrasound; PI-RADS, Prostate Imaging Reporting and Data System; PSA, prostate specific antigen; MRI, magnetic resonance imaging. 


\section{MRI sequences and biopsy techniques}

Multi-parametric MRI was performed prior to prostate biopsy and the MRI sequences included T2-weighted, diffusionweighted, and dynamic contrast-enhanced axial magnetic resonance (MR) images according to the guidelines of PI-RADSV2 [13]. A single radiologist evaluated MR images and categorized PIRADS 4 or 5 as an index lesion. All biopsies were performed by the same radiologist who had 17-year experience of genitourinary imaging and intervention.

First, target biopsy was performed in PI-RADS 4 and 5 if these categories were visible on TRUS. Then, systematic biopsy was added when tumor margin was not completely visible on TRUS. Determining whether or not the combination biopsy (target and systematic biopsies) was necessary simply depended on how TRUS operator subjectively assessed the lesion visibility on TRUS. He tried to add systematic biopsy as many as possible not to miss significant cancer in the prostate other than PI-RADS 4 or 5. Target cores were sampled within the PI-RADS 4 or 5 and then systematic cores were obtained from the other prostate tissue categorized as PI-RADS 1 or 2 .

The biopsy operator was familiar with the following TRUS techniques and imaging features [10]. First, fundamental imaging was used for lesion detection instead of harmonic imaging. Second, the dynamic range of the TRUS was kept at less than 50 to increase the tissue contrast. Third, the prostate was not compressed until PI-RADS 4 or 5 was detected. Fourth, a TRUS lesion was located more superiorly than an MRI lesion if it was closer to the posterior capsule (Fig. 2). Fifth, a TRUS lesion was located more inferiorly than an MRI lesion if it was closer to the anterior capsule (Fig. 3). Six, peripheral and transition cancers appeared to be hypoechoic and hyperechoic,
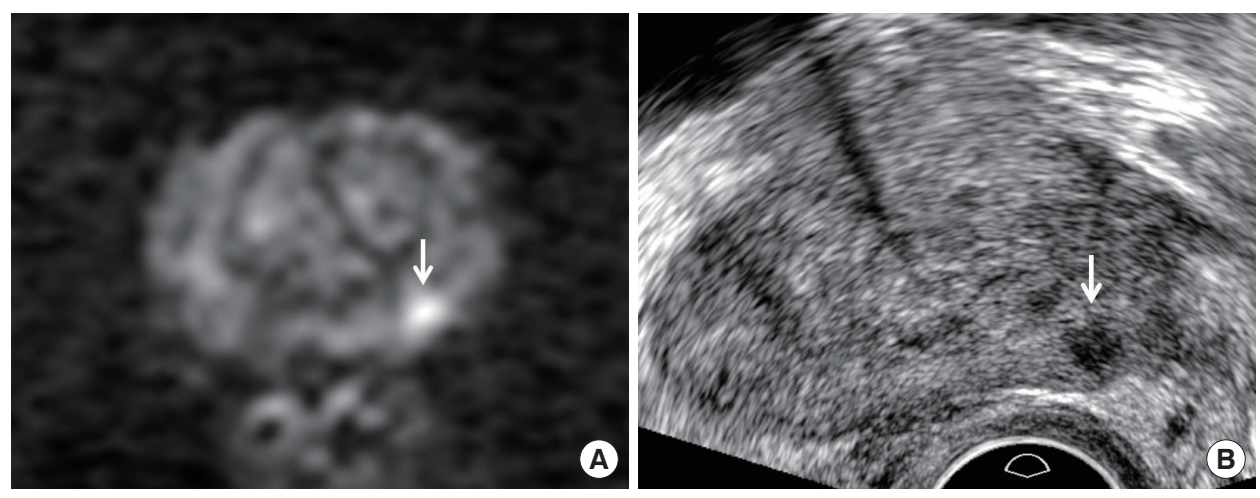

Fig. 2. A 64-year-old man with Prostate Imaging Reporting and Data System (PI-RADS) 4 peripheral cancer. (A) Diffusion-weighted axial magnetic resonance image shows a hyperintense mass (white arrow) in the left mid-gland peripheral zone. The lesion measures $5.3 \mathrm{~mm}$. His prostate specific antigen was $4.28 \mathrm{ng} / \mathrm{mL}$. (B) Transverse transrectal ultrasound (TRUS) image shows that the mass (white arrow) is hypoechoic and located in the left base peripheral zone. TRUS-guided target biopsy confirmed Gleason score $7(3+4)$ adenocarcinoma in all target cores $(n=4)$. TRUS-guided systematic biopsy did not detect prostate cancer.
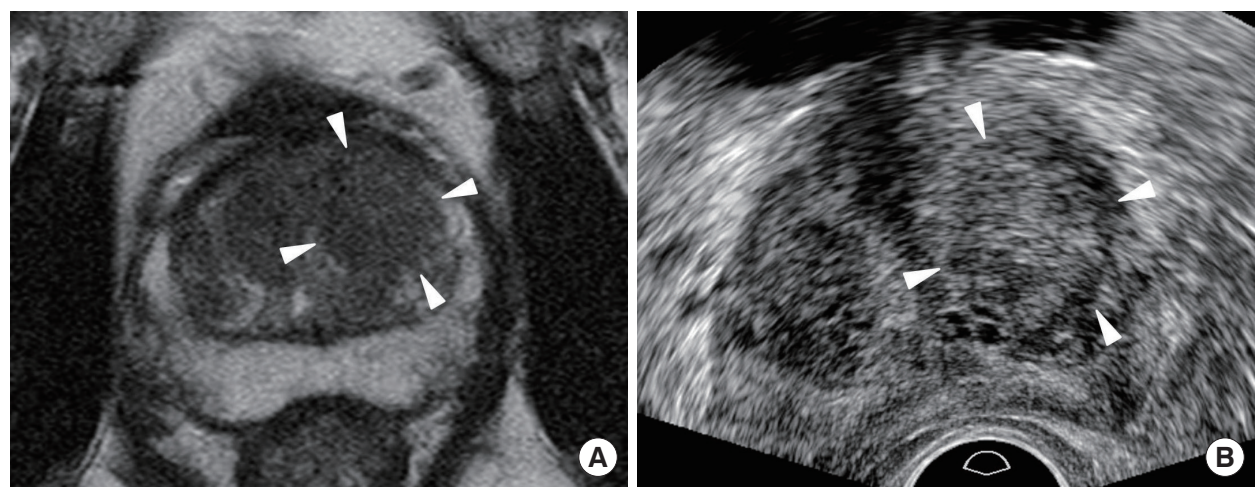

Fig. 3. A 69-year-old man with Prostate Imaging Reporting and Data System (PI-RADS) 5 transition cancer. (A) T2-weighted axial magnetic resonance image shows a hypointense mass (white arrowheads) in the left base transition zone. The lesion measures $15.4 \mathrm{~mm}$. His prostate specific antigen was $6.98 \mathrm{ng} / \mathrm{mL}$. (B) Transverse transrectal ultrasound (TRUS) image shows that the mass (white arrowheads) is hyperechoic and located in the left midgInad transition zone. TRUS-guided target biopsy confirmed Gleason score $7(4+3)$ adenocarcinoma in all target cores ( $n=4)$. TRUSguided systematic biopsy detected Gleason score $7(3+4)$ adenocarcinoma in two cores from sextant cores. 
respectively (Figs. 2, 3). Finally, higher-scoring PI-RADS lesions became more hypoechoic or hyperechoic (Figs. 2, 3). According to these TRUS techniques and imaging features, he already had performed TRUS-guided biopsy for targeting 432 PI-RADS 4 or 5 between March 2014 and May 2018.

\section{Data analysis}

MRI images were not reviewed at Picture Archiving and Communication System (PACS) station to re-categorize prostate lesions with PI-RADSV2. The original PI-RADS scores which were made by a radiologist prior to biopsy, were used for analysis.

Overall cancer detection rate (CDR) was calculated by the number of all cancer cases divided by the number of all biopsy cases in each group or each biopsy. Significant cancer was defined as one with Gleason score (GS) $\geq 7$. Significant CDR was calculated by the number of all GS $\geq 7$ cases divided by the number of all biopsy cases in each group or each biopsy. Overall and significant CDRs were compared between the groups or between the biopsies.

Positive core rate (PCR) was calculated by the number of all cancer cores divided by the number of all biopsy cores. PCRs were compared between the groups or between the biopsies.

The size of peripheral tumor was measured at diffusion-weighted axial images and that of transition tumor was measured at T2-weighted axial MR images. When a tumor involved both zones, the tumor size was measured depending on the dominant location. Therefore, when a tumor involved peripheral zone more dominantly than transition zone, the

Table 2. Comparison of biopsy outcomes between PI-RADS 4 and 5

\begin{tabular}{lcccc}
\hline \multirow{5}{*}{$\begin{array}{c}\text { Biopsy } \\
\text { outcome }\end{array}$} & $\begin{array}{c}\text { TRUS-guided biopsy } \\
(\mathrm{n}=94)\end{array}$ & & \\
\cline { 2 - 4 } & $\begin{array}{c}\text { PI-RADS 4 } \\
(\mathrm{n}=59)\end{array}$ & $\begin{array}{c}\text { PI-RADS 5 } \\
(\mathrm{n}=35)\end{array}$ & & \\
\hline Overall CDR (\%) & 57.6 & 94.3 & 12.132 & 0.0001 \\
& $(34 / 59)$ & $(33 / 35)$ & $(2.658-55.372)$ & \\
Significant & 44.1 & 85.7 & 7.615 & $<0.0001$ \\
CDR (\%) & $(26 / 59)$ & $(30 / 35)$ & $(2.593-22.370)$ & \\
PCR (\%) & 26.7 & 58.5 & 3.866 & $<0.0001$ \\
& $(188 / 703)$ & $(175 / 299)$ & $(2.909-5.138)$ & \\
Target to & $5: 54$ & $19: 16$ & 12.825 & $<0.0001$ \\
combination & & & $(4.133-39.797)$ & \\
biopsy ratio & & & & \\
\hline
\end{tabular}

PI-RADS, Prostate Imaging Reporting and Data System; TRUS, transrectal ultrasound; $\mathrm{OR}$, odds ratio; $\mathrm{Cl}$, confidence interval; $\mathrm{CDR}$, cancer detection rate; $\mathrm{PCR}$, positive core rate. tumor size was measured on diffusion-weighted MR images. The median numbers of all or cancer cores obtained by target biopsy were compared between the groups. The median GSs of the target biopsy were compared between the groups.

\section{Statistical analysis}

Mann-Whitney test was used to compare patient age, PSA, prostate volume, PSA density, MRI-biopsy interval, tumor size, biopsy core, and GS between the groups. Fisher's exact test was used to compare CDRs and PCRs between the groups or the biopsies. Odds ratio was obtained to quantify the strength of the association between the groups. The approximation of Woolf was used to calculate $95 \%$ confidence interval.

Commercially available software (PASW Statistics version 20.0, IBM Co., Armonk, NY, USA) was used for statistical analysis. A two-side $P$-value of less than 0.05 was considered statistically significant.

\section{Ethical statement}

This retrospective study was conducted on human and was approved by Institutional Review Board of Samsung Medical Center (IRB number: 2019-02-102-001). Informed consent was waived.

\section{RESULTS}

PI-RADS 4 group had younger age $(P=0.0002)$, lower PSA $(P=$ $0.0002)$, lower $P S A$ density $(P=0.0037)$, longer MRI-Biopsy interval $(P=0.0075)$, and smaller tumor size $(P<0.0001)$ than PIRADS 5 group (Table 1$)$. There was no difference between the groups in terms of prostate volume $(P=0.9813)$. The peripher-

Table 3. Comparison of target biopsy outcomes between the PIRADS 4 and 5

\begin{tabular}{|c|c|c|c|c|}
\hline \multirow{2}{*}{$\begin{array}{l}\text { Biopsy } \\
\text { outcome }\end{array}$} & \multicolumn{2}{|c|}{ Target biopsy $(n=94)$} & \multirow[b]{2}{*}{ OR $(95 \% \mathrm{Cl})$} & \multirow[b]{2}{*}{ P-value } \\
\hline & $\begin{array}{c}\text { PI-RADS } 4 \\
(n=59)\end{array}$ & $\begin{array}{c}\text { PI-RADS } 5 \\
(n=35)\end{array}$ & & \\
\hline $\begin{array}{l}\text { Overall CDR } \\
(\%)\end{array}$ & $\begin{array}{c}52.5 \\
(31 / 59)\end{array}$ & $\begin{array}{c}91.4 \\
(32 / 35)\end{array}$ & $\begin{array}{c}9.634 \\
(2.654-34.978)\end{array}$ & $<0.0001$ \\
\hline $\begin{array}{l}\text { Significant } \\
\text { CDR }(\%)\end{array}$ & $\begin{array}{c}42.4 \\
(25 / 59)\end{array}$ & $\begin{array}{c}82.6 \\
(29 / 35)\end{array}$ & $\begin{array}{c}6.573 \\
(2.371-18.224)\end{array}$ & 0.0002 \\
\hline PCR (\%) & $\begin{array}{c}44.0 \\
(159 / 361)\end{array}$ & $\begin{array}{c}77.7 \\
(157 / 202)\end{array}$ & $\begin{array}{c}4.432 \\
(2.997-6.555)\end{array}$ & $<0.0001$ \\
\hline
\end{tabular}

PI-RADS, Prostate Imaging Reporting and Data System; OR, odds ratio; $\mathrm{Cl}$, confidence interval; $\mathrm{CDR}$, cancer detection rate; $\mathrm{PCR}$, positive core rate. 
Table 4. Comparison of systematic biopsy outcomes between PIRADS 4 and 5

\begin{tabular}{|c|c|c|c|c|}
\hline \multirow{2}{*}{$\begin{array}{l}\text { Biopsy } \\
\text { outcome }\end{array}$} & \multicolumn{2}{|c|}{$\begin{array}{l}\text { Systematic biopsy } \\
\qquad(n=70)\end{array}$} & \multirow{2}{*}{ OR $(95 \% \mathrm{Cl})$} & \multirow{2}{*}{ P-value } \\
\hline & $\begin{array}{c}\text { PI-RADS } 4 \\
(n=54)\end{array}$ & $\begin{array}{c}\text { PI-RADS } 5 \\
(n=16)\end{array}$ & & \\
\hline $\begin{array}{l}\text { Overall CDR } \\
(\%)\end{array}$ & $\begin{array}{c}31.5 \\
(17 / 54)\end{array}$ & $\begin{array}{c}50.0 \\
(8 / 16)\end{array}$ & $\begin{array}{c}2.176 \\
(0.6988-6.779)\end{array}$ & 0.2363 \\
\hline $\begin{array}{l}\text { Significant } \\
\text { CDR }(\%)\end{array}$ & $\begin{array}{c}13.0 \\
(7 / 54)\end{array}$ & $\begin{array}{c}25.0 \\
(4 / 16)\end{array}$ & $\begin{array}{c}2.238 \\
(0.5615-8.921)\end{array}$ & 0.2589 \\
\hline PCR (\%) & $\begin{array}{c}8.5 \\
(29 / 342)\end{array}$ & $\begin{array}{c}18.6 \\
(18 / 97)\end{array}$ & $\begin{array}{c}2.459 \\
(1.299-4.654)\end{array}$ & 0.0083 \\
\hline
\end{tabular}

PI-RADS, Prostate Imaging Reporting and Data System; OR, odds ratio; $\mathrm{Cl}$, confidence interval; $\mathrm{CDR}$, cancer detection rate; $\mathrm{PCR}$, positive core rate.

al to transition lesion ratio was 56:3 in the PI-RADS 4 group and 17:18 in the PI-RADS 5 group $(\mathrm{P}<0.0001)$. The peripheral to transition cancer ratio was 31:3 in the PI-RADS 4 group and 16:17 in the PI-RADS 5 group $(P=0.0002)$. The median number of target cores was 6 ( 2 to 11 ) in the PI-RADS 4 group and 6 (2 to 12$)$ in the PI-RADS 5 group $(P=0.5089)$. The median number of target cancer cores was 5 ( 0 to 10$)$ in the PI-RADS 4 group and 5 ( 0 to 9$)$ in the PI-RADS 5 group $(P=0.9498)$. The median GS was 7 (6 to 8) in the PI-RADS 4 group and 7 (6 to 8) in the PI-RADS 5 group $(P=0.0750)$.

The overall CDR was $57.6 \%$ (34/59) in the PI-RADS 4 group and $94.3 \%(33 / 35)$ in the PI-RADS 5 group $(P=0.0001)$ (Table 2). The significant CDR was $44.1 \%$ (26/59) in the PI-RADS 4 group and $85.7 \%(30 / 35)$ in the PI-RADS 5 group $(P<0.0001)$. The PCR was $26.7 \%(188 / 703)$ in the PI-RADS 4 group and $58.5 \%(175 / 299)$ in the PI-RADS 5 group $(P<0.0001)$. The target to combination biopsy ratio was 5:54 in the PI-RADS 4 group and 19:16 in the PI-RADS 5 group $(P<0.0001)$.

The overall CDR of the target biopsy was $52.5 \%(31 / 59)$ in the PI-RADS 4 group and $91.4 \%(32 / 35)$ in the PI-RADS 5 group $(P<0.0001)$ (Table 3$)$. The significant $C D R$ of the target biopsy were $42.4 \%(25 / 59)$ in the PI-RADS 4 group and $82.6 \%$ $(29 / 35)$ in the PI-RADS 5 group $(P=0.0002)$. The PCR of the target biopsy were $44.0 \%(159 / 361)$ in the PI-RADS 4 group and $77.7 \%(157 / 202)$ in the PI-RADS 5 group $(P<0.0001)$.

The overall CDR of the systematic biopsy was $31.5 \%$ $(17 / 54)$ in the PI-RADS 4 group and $50.0 \%(8 / 16)$ in the PIRADS 5 group ( $P=0.2363$ ) (Table 4). The significant CDR of the systematic biopsy was $13.0 \%(7 / 54)$ in the PI-RADS 4 group and $25.0 \%(4 / 16)$ in the PI-RADS 5 group $(P=0.2589)$. The PCR of the systematic biopsy was $8.5 \%(29 / 342)$ in the
Table 5. Comparison of combination biopsy outcomes between PI-RADS 4 and 5

\begin{tabular}{|c|c|c|c|c|}
\hline \multirow{2}{*}{$\begin{array}{l}\text { Biopsy } \\
\text { outcome }\end{array}$} & \multicolumn{2}{|c|}{$\begin{array}{l}\text { Combination biopsy } \\
\qquad(n=70)\end{array}$} & \multirow{2}{*}{ OR $(95 \% \mathrm{Cl})$} & \multirow{2}{*}{ P-value } \\
\hline & $\begin{array}{l}\text { PI-RADS } 4 \\
(n=54)\end{array}$ & $\begin{array}{l}\text { PI-RADS } 5 \\
(n=16)\end{array}$ & & \\
\hline $\begin{array}{l}\text { Overall CDR } \\
(\%)\end{array}$ & $\begin{array}{c}53.7 \\
(29 / 54)\end{array}$ & $\begin{array}{c}87.5 \\
(14 / 16)\end{array}$ & $\begin{array}{c}6.034 \\
(1.248-26.168)\end{array}$ & 0.0189 \\
\hline $\begin{array}{l}\text { Significant } \\
\text { CDR (\%) }\end{array}$ & $\begin{array}{c}38.9 \\
(21 / 54)\end{array}$ & $\begin{array}{c}81.3 \\
(13 / 16)\end{array}$ & $\begin{array}{c}6.810 \\
(1.731-26.791)\end{array}$ & 0.0040 \\
\hline PCR (\%) & $\begin{array}{c}22.4 \\
(149 / 664)\end{array}$ & $\begin{array}{c}43.5 \\
(84 / 193)\end{array}$ & $\begin{array}{c}2.664 \\
(1.900-3.735)\end{array}$ & $<0.0001$ \\
\hline
\end{tabular}

PI-RADS, Prostate Imaging Reporting and Data System; OR, odds ratio; $\mathrm{Cl}$, confidence interval; $\mathrm{CDR}$, cancer detection rate; $\mathrm{PCR}$, positive core rate.

PI-RADS 4 group and 18.6\% (18/97) in the PI-RADS 5 group $(P=0.0083)$.

The overall CDR of the combination biopsy was $53.7 \%$ $(29 / 54)$ in the PI-RADS 4 group and $87.5 \%(14 / 16)$ in the PIRADS 5 group $(P=0.0189)$ (Table 5). The significant CDR of the combination biopsy was $38.9 \%(21 / 54)$ in the PI-RADS 4 group and $81.3 \%(13 / 16)$ in the PI-RADS 5 group $(P=0.0040)$. The PCR of the combination biopsy was $22.4 \%(149 / 664)$ in the PI-RADS 4 group and $43.5 \%(84 / 193)$ in the PI-RADS 5 group $(P<0.0001)$.

Of 34 cancer-proven PI-RADS 4 cases, 14 were diagnosed by both, 17 by target biopsy (Fig. 2), and 3 by systematic biopsy. The three cancers detected by systematic biopsy alone were all GS 6 tumors. Systematic biopsy detected a GS 7 tumor which was underscored as GS 6 tumor by target biopsy. Of 33 cancer-proven PI-RADS 5 cases, seven were diagnosed by both (Fig. 3), 25 by target biopsy, and one by systematic biopsy. The significant cancer detected by systematic biopsy alone was a GS 7 tumor.

\section{DISCUSSION}

PI-RADS 4 and 5 have a higher risk of significant cancer than PI-RADS 1-3. As a prostate cancer becomes GS 7 or higher, the tumor has stronger signal intensity on diffusion-weighted or T2-weighted MR images. Target biopsy is performed in PI-RADS 4 or 5 under MRI-TRUS fusion or in-bore MRI guidance because this category is clearly visible on MRI. In contrast, TRUS has not been concerned in targeting a prostate cancer because this modality cannot clearly depict it. Interestingly, it is not uncommon for an experienced operator to 
see a well-demarcated tumor on TRUS. This tumor frequently turns out to be a significant cancer. Besides, it is still unclear if PI-RADS 4 or 5 is visible on TRUS or if this category is targeted by TRUS. Our study showed that the significant CDRs of PI-RADS 4 and 5 using TRUS is not inferior to those using MRI-TRUS fusion or in-bore MRI in previously published investigations. They reported that the significant CDRs of PIRADS 4 and 5 are $22.1 \%$ to $78.0 \%$ and $72.4 \%$ to $90.7 \%$, respectively $[4-6,8,14-16]$. Our significant CDRs of these categories were $44.1 \%$ and $85.7 \%$, respectively.

New TRUS techniques were applied in our study. One is that prostate was not compressed until a lesion was detected. If prostate is compressed, a cancer is likely to be compressed so that it cannot be seen on TRUS. A small peripheral cancer near Denonvilliers' fascia becomes flat frequently due to prostate compression. It is difficult to differentiate the compressed hypoechoic tumor from the thick hypoechoic rectoprostatic fascia. The other is that tumor location on TRUS looked quite different from that on MRI. The axial TRUS images are obtained obliquely to urethra, whereas the axial MR images are done perpendicular to urethra. For the same reason, the tumor size or shape on TRUS also appears different from that on MRI. The difference of MRI-TRUS scan axis makes it difficult for TRUS operators to find a lesion that is categorized as PI-RADS 4 or 5 lesion on MRI.

New TRUS features of significant cancer were also applied in our study. It is generally accepted that prostate cancer is not clearly depicted on TRUS. However, peripheral cancer becomes more hypoechoic on TRUS if a GS becomes 7 or higher. Our study demonstrated that the CDR of peripheral PI-RADS 4 or 5 was $100 \%$ with target biopsy alone when the tumor was completely demarcated on TRUS. Unfortunately, the TRUS features of transition cancer are not well known. Our study assumed that transition cancer becomes hyperechoic if GS increases. Investigations reporting the TRUS features of transition cancer are extremely rare. Several studies [17-19] reported the presence of hyperechoic prostate cancers, but they did not demonstrate that these tumors are transition cancers. Some investigations have reported that transition cancer is more hyperechoic than peripheral cancer [10-13]. Our study showed that the CDR of transition PI-RADS 4 or 5 were higher than that of peripheral PI-RADS 4 or 5 using the new TRUS features of transition tumor. Further investigation is necessary to validate the TRUS feature of transition tumor because the number of transition tumor was small. Recently, ultra-high resolution TRUS probe has developed and provided a potential to image or biopsy a signifi- cant prostate cancer. Accordingly, it will be promising for improving lesion detection and sampling under the guidance [20-22]. Recently, much higher-resolution TRUS has been introduced to image and biopsy a prostate cancer. Increasing resolution may lead to increasing more precise detection or biopsy of a PI-RADS 4 or 5 lesion [20,21,23].

Systematic biopsy was frequently performed in our study even if PI-RADS 4 or 5 was all visible on TRUS. The lesion visibility was different each other. Systematic biopsy was added to target biopsy when an operator was not convinced that tumor margin was completely visible on TRUS. The overall and significant CDR had been improved approximately $10 \%$ to $20 \%$ by means of increasing the number of systematic biopsies before. Systematic biopsy yielded $13 \%$ to $25 \%$ significant CDR in the prostate other than PI-RADS 4 or 5 in our study. These CDRs are much lower than those of target biopsy in PI-RADS 4 or 5 . These findings indicate that prostate cancer is frequently multifocal [24-26]. Therefore, insignificant cancer may be detected by target biopsy whereas significant cancer may be done by systematic biopsy. If target biopsy alone is performed using MRI-TRUS fusion or in-bore $M R I$, significant cancer will be missed in the prostate other than PI-RADS 4 or 5. Indeed, systematic biopsy is necessary in PI-RADS 4 or 5 if unilateral focal therapy is planned for management. Furthermore, systematic biopsy may provide additional value to target biopsy in case that PI-RADS 4 or 5 is mis-targeted or inflammation [27].

Our study had some limitations. First, there were no objective criteria for adding systematic biopsy to target biopsy. The lesion visibility is a key finding to determine whether or not systematic biopsy is performed. However, it relies on operator's skill and experience. Second, the optimal protocol of systematic biopsy was not established in PI-RADS 4 or 5 . Increasing number of systematic cores increases not only significant $C D R$, but also complication rate such as pain, urinary retention, bleeding, and infection [28-31]. Third, the optimal number of target cores was not established either. When GS 4 component is small and eccentric in PI-RADS 4 or 5 , target biopsy only obtained from the tumor center may miss significant cancer. Our study sampled target cores from peripheral area as well as central area in the PI-RADS 4 or 5 , subsequently resulting in increased number of target cores. Fourth, still, TRUS-guided target biopsy is not widely accepted as a biopsy technique. Fifth, the number of cases was relatively small. Last, our study design was retrospective.

In conclusion, new TRUS biopsy techniques provide a good significant CDR in patients with PI-RADS 4 and 5 if an 
operator is familiar with the new TRUS techniques and imaging features. However, systematic biopsy is difficult to skip because it can detect additional cancers which are missed or underscored by target biopsy. When these categories are identified on pre-biopsy MRI, TRUS should be first performed to assess lesion visibility prior to MRI-TRUS fusion or in-bore MRI-guided biopsy.

\section{CONFLICTS OF INTEREST}

No potential conflict of interest relevant to this article was reported.

\section{ORCID}

Taein An https://orcid.org/0000-0002-0569-6022

Byung Kwan Park https://orcid.org/0000-0002-4114-8859

\section{AUTHOR CONTRIBUTIONS}

Conception or design: TA, BKP.

Acquisition, analysis, or interpretation of data: TA, BKP.

Drafting the work or revising: TA, BKP.

Final approval of the manuscript: TA, BKP.

\section{REFERENCES}

1. Weinreb JC, Barentsz JO, Choyke PL, Cornud F, Haider MA, Macura KJ, et al. PI-RADS prostate imaging: reporting and data system: 2015, Version 2. Eur Urol 2016;69:16-40.

2. Barentsz JO, Weinreb JC, Verma S, Thoeny HC, Tempany CM, Shtern F, et al. Synopsis of the PI-RADS v2 guidelines for multiparametric prostate magnetic resonance imaging and recommendations for use. Eur Urol 2016;69:41-9.

3. Purysko AS, Rosenkrantz AB, Barentsz JO, Weinreb JC, Macura KJ. PI-RADS version 2: a pictorial update. Radiographics 2016;36:1354-72.

4. Greer MD, Shih JH, Lay N, Barrett T, Kayat Bittencourt L, Borofsky $\mathrm{S}$, et al. Validation of the dominant sequence paradigm and role of dynamic contrast-enhanced imaging in PI-RADS version 2. Radiology 2017;285:859-69.

5. Hofbauer SL, Maxeiner A, Kittner B, Heckmann R, Reimann $\mathrm{M}$, Wiemer $\mathrm{L}$, et al. Validation of prostate imaging reporting and data system version 2 for the detection of prostate cancer. J Urol 2018;200:767-73.

6. Mehralivand S, Bednarova S, Shih JH, Mertan FV, Gaur S, Merino MJ, et al. Prospective evaluation of PI-RADS ${ }^{\mathrm{TM}}$ ver- sion 2 using the International Society of Urological Pathology prostate cancer grade group system. J Urol 2017; 198:583-90.

7. Park SY, Jung DC, Oh YT, Cho NH, Choi YD, Rha KH, et al. Prostate cancer: PI-RADS version 2 helps preoperatively predict clinically significant cancers. Radiology 2016;280: 108-16.

8. Tan N, Lin WC, Khoshnoodi P, Asvadi NH, Yoshida J, Margolis DJ, et al. In-Bore 3-T MR-guided transrectal targeted prostate biopsy: prostate imaging reporting and data system version 2-based diagnostic performance for detection of prostate cancer. Radiology 2017;283:130-9.

9. Thai JN, Narayanan HA, George AK, Siddiqui MM, Shah P, Mertan FV, et al. Validation of PI-RADS version 2 in transition zone lesions for the detection of prostate cancer. Radiology 2018;288:485-91.

10. Park BK, Park SY. New biopsy techniques and imaging features of transrectal ultrasound for targeting PI-RADS 4 and 5 lesions. J Clin Med 2020;9:530.

11. Park BK. Ultrasound-guided genitourinary interventions: principles and techniques. Ultrasonography 2017;36: 336-48.

12. Park BK. Image-guided prostate biopsy: necessity for terminology standardization. J Ultrasound Med 2020;39: 191-6.

13. Park SY, Park BK. Necessity of differentiating small $(<10$ $\mathrm{mm}$ ) and large ( $\geq 10 \mathrm{~mm}$ ) PI-RADS 4. World J Urol 2020; 38:1473-9.

14. Kasivisvanathan V, Rannikko AS, Borghi M, Panebianco V, Mynderse LA, Vaarala MH, et al. MRI-targeted or standard biopsy for prostate-cancer diagnosis. N Engl J Med 2018; 378:1767-77.

15. Cash H, Maxeiner A, Stephan C, Fischer T, Durmus T, Holzmann J, et al. The detection of significant prostate cancer is correlated with the Prostate Imaging Reporting and Data System (PI-RADS) in MRI/transrectal ultrasound fusion biopsy. World J Urol 2016;34:525-32.

16. Venderink $W$, van der Leest $M$, van Luijtelaar $A$, van de Ven WJM, Futterer JJ, Sedelaar JPM, et al. Retrospective comparison of direct in-bore magnetic resonance imaging (MRI)-guided biopsy and fusion-guided biopsy in patients with MRI lesions which are likely or highly likely to be clinically significant prostate cancer. World J Urol 2017; 35:1849-55.

17. Salo JO, Rannikko S, Makinen J, Lehtonen T. Echogenic structure of prostatic cancer imaged on radical prostatectomy specimens. Prostate 1987;10:1-9. 
18. Egawa S, Wheeler TM, Greene DR, Scardino PT. Unusual hyperechoic appearance of prostate cancer on transrectal ultrasonography. Br J Urol 1992;69:169-74.

19. Spajic B, Eupic H, Tomas D, Stimac G, Kruslin B, Kraus O. The incidence of hyperechoic prostate cancer in transrectal ultrasound-guided biopsy specimens. Urology 2007; 70:734-7.

20. Ghai S, Eure G, Fradet V, Hyndman ME, McGrath T, Wodlinger $\mathrm{B}$, et al. Assessing cancer risk on novel $29 \mathrm{MHz}$ micro-ultrasound images of the prostate: creation of the micro-ultrasound protocol for prostate risk identification. J Urol 2016;196:562-9.

21. Rohrbach D, Wodlinger B, Wen J, Mamou J, Feleppa E. High-frequency quantitative ultrasound for imaging prostate cancer using a novel micro-ultrasound scanner. ULtrasound Med Biol 2018;44:1341-54.

22. Tokas T, Grabski B, Paul U, Baurle L, Loch T. A 12-year follow-up of ANNA/C-TRUS image-targeted biopsies in patients suspicious for prostate cancer. World J Urol 2018; 36:699-704.

23. Eure G, Fanney D, Lin J, Wodlinger B, Ghai S. Comparison of conventional transrectal ultrasound, magnetic resonance imaging, and micro-ultrasound for visualizing prostate cancer in an active surveillance population: a feasibility study. Can Urol Assoc J 2019;13:E70-7.

24. Djavan B, Susani M, Bursa B, Basharkhah A, Simak R, Marberger M. Predictability and significance of multifocal prostate cancer in the radical prostatectomy specimen. Tech Urol 1999;5:139-42.

25. Andreoiu M, Cheng L. Multifocal prostate cancer: biologic, prognostic, and therapeutic implications. Hum Pathol 2010;41:781-93.

26. Wise AM, Stamey TA, McNeal JE, Clayton JL. Morphologic and clinical significance of multifocal prostate cancers in radical prostatectomy specimens. Urology 2002;60:264-9.

27. Meier-Schroers M, Kukuk G, Wolter K, Decker G, Fischer S, Marx C, et al. Differentiation of prostatitis and prostate cancer using the Prostate Imaging-Reporting and Data System (PI-RADS). Eur J Radiol 2016;85:1304-11.

28. Nam RK, Saskin R, Lee Y, Liu Y, Law C, Klotz LH, et al. Increasing hospital admission rates for urological complications after transrectal ultrasound guided prostate biopsy. J Urol 2010;183:963-8.

29. Loeb S, Carter HB, Berndt SI, Ricker W, Schaeffer EM. Complications after prostate biopsy: data from SEER-Medicare. J Urol 2011;186:1830-4.

30. Loeb S, Vellekoop A, Ahmed HU, Catto J, Emberton M, Nam R, et al. Systematic review of complications of prostate biopsy. Eur Urol 2013;64:876-92.

31. Roberts MJ, Bennett HY, Harris PN, Holmes M, Grummet J, Naber K, et al. Prostate biopsy-related infection: a systematic review of risk factors, prevention strategies, and management approaches. Urology 2017;104:11-21. 